\title{
Le pari ambitieux du cluster maritime du Québec : état de la situation et politiques publiques'
}

\section{The ambitious wager of Quebec's maritime cluster: current situation and public policies}

\author{
David Doloreux ${ }^{\mathrm{a}}$, Yannik Melançon ${ }^{\mathrm{b}}$
}

${ }^{a}$ Chaire de recherche sur la francophonie canadienne, École de Gestion, Université d'Ottawa, Ottawa, Ontario, K1N 6N5 Canada

${ }^{b}$ Département des Sciences Humaines, Université du Québec à Rimouski, 300 allée des Ursulines, Rimouski, Québec, G5L 3Al Canada

\section{Résumé}

Cet article est consacré à l'étude de cas du cluster maritime du Québec. Il discute des facteurs qui expliquent pourquoi les activités innovantes n'ont pas émergé dans le secteur maritime considéré au départ comme étant porteur pour la croissance industrielle et économique de la région du Québec maritime. Après avoir brièvement décrit le contexte et le développement du cluster maritime, nous discutons de certains facteurs qui pourraient expliquer l'activité innovante globale faible des entreprises maritimes et l'absence d'une masse critique d'acteurs pour faire émerger des dynamiques propres aux clusters. Des propositions générales de politiques sont présentées en conclusion.

(C) 2006 Lavoisier, Paris. Tous droits réservés.

1 David Doloreux remercie le Fonds québécois de la recherche sur la société et la culture (FQRSC) pour le soutien financier du projet. Il tient également à remercier Ludovic Lévesque, Jacques Paquin, Maryse Langevin et Luc Desaulniers pour les commentaires apportés pour l'amélioration de l'article. Les propos exprimés dans cet article n'engagent que les auteurs.

Adresses email : Doloreux@management.uottawa.ca•Yannik.Melancon@uqar.qc.ca 


\section{Summary}

This article discusses the case-study of the Quebec's maritime cluster. It discusses the factors that explain why significant innovation activities in maritime industry did not emerge in what might have been considered at the outset a promising area of growth of this industry and the Quebec maritime region. After briefly describing the development of the maritime cluster, we discuss some factors that might explain the weak performance of the maritime industry in terms of innovation activities and the lack of critical mass of actors to foster clustering dynamics. A range of general policies issues are discussed in the conclusion.

(C) 2006 Lavoisier, Paris. Tous droits réservés.

Mots-clés : cluster maritime, Québec, entreprises, système de soutien, politiques publiques

Keywords: maritime cluster, Quebec, enterprises, support system, public policies

\section{Introduction}

Aujourd'hui, des études comparant les clusters mis en place dans diverses régions illustrent à quel point les politiques visant à favoriser l'émergence et le développement de clusters régionaux sont incontournables et ce, tant dans les régions urbaines que périphériques, tant dans les industries de haute technologie que dans celles à faible intensité technologique ou de services (IRE, 2006; Porter, 2004; European Commission, 2003; OECD, 2001).

Les politiques de développement régional québécoises et canadiennes témoignent elles aussi de cet engouement pour le développement des clusters. Ainsi, dans Atteindre l'excellence - la stratégie d'innovation du Canada (2002), le Gouvernement fédéral met en place une politique visant notamment à renforcer l'innovation régionale en soutenant le développement de clusters concurrentiels à l'échelle internationale, principalement dans une dizaine de secteurs d'activités. S'inscrivant dans ces orientations nationales, le Conseil national de recherche du Canada a instauré dans les 12 régions où il est présent des mesures destinées à améliorer les retombées locales de savoirs, le tout dans le but de favoriser la création et la croissance de clusters régionaux (Conseil national de recherche du Canada, 2005).

C'est aussi dans les suites de la Stratégie d'innovation du Canada que s'inscrit le programme des Initiatives régionales stratégiques de Développement économique Canada, l'agence fédérale responsable de promouvoir le développement économique des régions du Québec. En effet, ce programme contribue au financement d'initiatives régionales structurantes dans un nombre limité de clusters ciblés dans chacune des régions du Québec. Le projet d'Action concertée de coopération régionale de développement $(A C C O R D)$ est quant à lui l'outil défini par le Gouvernement du Québec pour contribuer au développement de clusters régionaux. ACCORD adopte une approche participative au sein de laquelle, dans chacune des régions administratives du Québec, les principaux acteurs régionaux ont été invités à identifier des créneaux jugés porteurs pour le développement économique de leur région et à travailler collectivement au développement de ceux-ci. Afin de soutenir les créneaux identifiés dans ACCORD, des politiques et programmes sectoriels ont été instaurés dans les régions québécoises.

Parmi les clusters régionaux identifiés par les politiques publiques comme enjeu de développement prioritaire figure le cluster maritime du Québec. Cet article est consacré à l'étude 
de cas de ce cluster spécifique. Il discute des facteurs qui expliquent pourquoi les activités innovantes n'ont pas émergé dans le secteur maritime considéré au départ comme étant porteur pour la croissance industrielle et économique de la région du Québec maritime. Après avoir brièvement décrit le contexte et le développement du cluster maritime, nous discutons de certains facteurs qui pourraient expliquer l'activité innovante globale faible des entreprises maritimes et l'absence d'une masse critique d'acteurs pour faire émerger des dynamiques propres aux clusters. Des propositions générales de politiques sont présentées en conclusion.

\section{Contexte : le développement du cluster maritime du Québec}

Le Québec maritime n'est pas une région administrative, mais une région construite située dans le nord-est de la province de Québec et définie par rapport aux régions côtières de l'estuaire du Saint-Laurent. Le Québec maritime regroupe trois régions administratives (le Bas-SaintLaurent, la Gaspésie-Îles-de-la-Madeleine et la Côte-Nord) et s'étend sur près de 3600 kilomètres de côtes. Sa superficie est de $451880 \mathrm{~km}^{2}$, sa population est de 395562 personnes, pour une densité de population de $0,9 \mathrm{hab} . / \mathrm{km}^{2}$. Ses principaux centres urbains sont Rimouski, Rivièredu-Loup, Gaspé, Baie-Comeau et Sept-Îles. Le Québec maritime peut être considéré à bien des égards comme la région la plus périphérique au Québec. Pour ce qui est des principaux indicateurs économiques et de l'économie du savoir (croissance d'emplois, revenus, niveaux d'éducation), les régions du Québec maritime sont en retard par rapport au reste du Québec, exception faite des agglomérations urbaines du Bas-Saint-Laurent (Polèse et Shearmur, 2002).

L'émergence et le développement du cluster maritime du Québec ont été affectés par deux principaux éléments : (i) la présence d'infrastructures et d'institutions publiques et (ii) les politiques publiques.

Les activités du cluster maritime dans la région sont ancrées territorialement et peuvent être liées au développement des infrastructures maritimes entamé au tournant du XIX ${ }^{\mathrm{e}}$ siècle autour de Rimouski : installation d'un maréomètre en 1894, construction d'un quai de 800 pieds de long de 1901 à 1905, création en 1905 d'une station de pilotage par le ministère de la Marine, implantation d'un réseau de télégraphie sans fil en 1907, construction d'un phare en 1909.

Le développement du secteur maritime à Rimouski se poursuit avec la création en 1944 de l'Institut maritime du Québec, un centre technique de formation, et la création d'un programme de maîtrise en océanographie à l'Université du Québec à Rimouski (UQAR) en 1972. Historiquement, le développement de l'axe des sciences de la mer à l'UQAR a démarré avec la création de ce programme de maîtrise, et il s'est poursuivi avec la fondation d'une nouvelle institution de recherche en océanologie (INRSOcéanologie) en 1972, d'un département d'océanographie (1978), d'un programme de doctorat (1983), et du regroupement des chercheurs de l'UQAR et de l'INRS au sein d'un nouvel Institut des sciences de la mer (1999). En 1987, la fondation de l'Institut Maurice-Lamontagne, un des douze centres de recherche de Pêches et Océans Canada, a permis d'accroître significativement les activités de recherche et de renforcer la base institutionnelle du secteur maritime en offrant une expertise de recherche dans les domaines halieutiques, des sciences océaniques et de l'environnement.

Malgré tous ces développements institutionnels, les premiers signes visibles de la création du cluster maritime viennent cependant entre 1998 et 2002 avec une série 
d'investissements du secteur public dans les établissements de recherche et d'enseignement, de même que dans la promotion de l'innovation. Le but de ces investissements est de stimuler la compétitivité et l'innovation dans les régions du Québec maritime et d'accroître la base de connaissance et l'infrastructure de soutien au développement de l'industrie maritime de ces régions. Derrière la mise en place du cluster maritime se trouve la volonté des différents gouvernements de favoriser le développement d'un créneau d'excellence en sciences et technologies marines, ce qui implique d'accroître la masse critique d'entreprises et d'organisation de soutien, le tout afin de stimuler le développement de l'industrie maritime et de la rendre plus compétitive. Plusieurs politiques et programmes ont été initiés par les gouvernements dans le but de renverser les tendances lourdes qui affectaient les économies du Québec maritime et de mettre en valeur les ressources maritimes. Parmi les principales politiques ayant influencé l'émergence du cluster maritime, il y a l'Initiative régionale stratégique, la Planification stratégique régionale, Plein Cap sur la Mer et ACCORD.

Cependant, l'émergence du cluster maritime et la création d'une base institutionnelle dédiée à cette industrie n'auraient pas vu le jour sans la participation financière du Gouvernement fédéral par l'entremise de son programme Initiative régionale stratégique (IRS). La politique de l'IRS vise à augmenter, entre autres, la capacité technologique régionale dans le but de favoriser l'utilisation de technologies plus appropriées par les entreprises. Un autre objectif consiste à contribuer à la réalisation d'initiatives ayant un impact significatif sur le développement et la compétitivité de secteurs porteurs dans certaines régions. Dans le cas du cluster maritime, ce programme a entraîné des investissements de plus de 65 millions de dollars; ceux-ci ont contribué à la création de plusieurs organismes, soit: le Centre interdisciplinaire de développement en cartographie des océans, le Centre de recherche sur les biotechnologies marines, Technopole maritime du Québec, Innovation maritime et Biotechnologies Océanova.

L'histoire récente du cluster maritime du Québec constitue un exemple canadien de cluster dont la création est liée à la mise en place d'un ensemble de politiques et programmes stratégiques s'appuyant sur une spécialisation économique, des infrastructures et des institutions préexistantes. Les acteurs publics ont cherché à redynamiser ce tissu économique traditionnel et à créer des conditions favorables au développement de l'industrie maritime à travers des investissements directs qui visaient à accroître la base technologique régionale dédiée à celle-ci.

\section{La caractérisation du cluster maritime}

Le processus de recherche et de consultation mené dans le cadre de ce projet de recherche a permis de caractériser le cluster maritime du Québec (voir Doloreux, 2006; Doloreux et Shearmur, 2006). Nous pouvons résumer les principales caractéristiques du cluster maritime du Québec comme suit :

1.Le cluster maritime du Québec couvre une large gamme d'activités et est constitué de différents sous-secteurs d'activités tels que l'aquaculture, les pêches et la transformation des produits de la mer, la construction navale, les équipements maritimes, les sciences et technologies marines, et les autres activités liées au maritime. Les entreprises 
associées à ces secteurs d'activités présentent un niveau élevé de diversification, fabricant des produits dédiés à différents marchés, tels que l'alimentation, le transport, les technologies de l'information, la pharmaceutique, le biomédical ainsi que la cosmétique.

2. Le cluster maritime du Québec peut être considéré comme étant petit puisqu'il comprend 96 entreprises et 7563 travailleurs, ce qui représente approximativement 4,3\% des emplois de l'ensemble du Québec maritime.

3.Les entreprises du secteur de l'aquaculture, de même que celle des pêches et de la transformation des produits de la mer sont fortement concentrées dans la région de la Gaspésie-Îles-de-la-Madeleine alors que les entreprises des sciences et technologies marines sont principalement concentrées dans la région du Bas-Saint-Laurent, et plus particulièrement dans la ville de Rimouski. À l'exception de ces deux secteurs d'activité, l'industrie maritime se répartit de façon plutôt équilibrée dans les régions du Bas-SaintLaurent et de la Gaspésie-Îles-de-la-Madeleine alors que la Côte-Nord concentre un faible nombre d'entreprises et d'emplois.

4.Le développement des entreprises maritimes actuellement en opération dans les régions du Québec maritime est un phénomène relativement récent. Bien qu'un nombre important d'entreprises existantes ait démarré leurs activités durant les années 1980, près de la majorité d'entre elles a été créée après 1990. C'est précisément durant cette période que la création d'entreprises est la plus marquée, notamment pour ce qui est des entreprises des sciences et technologies marines. Il est important de noter la faible croissance de l'industrie après la période débutant en 2000.

5.Le cluster maritime du Québec est dominé par des micros et des petites entreprises. Plus de la moitié des entreprises comptent moins de 20 employés; cette proportion est encore plus élevée si nous considérons l'ensemble des entreprises de moins 100 employés (79,2\% des entreprises). Les entreprises de grande taille ne sont pas nombreuses et sont principalement actives dans les secteurs de la construction navale et de la transformation des produits marins.

\section{Le diagnostic : la performance du cluster maritime}

\subsection{La compétitivité des entreprises maritimes}

Selon Porter $(2004$; 2003), un des objectifs centraux dans la mise en place de clusters régionaux est de stimuler les activités à forte valeur ajoutée grâce à l'innovation, la coopération, et les synergies que les entreprises développeront avec les autres membres du cluster. Parmi l'ensemble des travaux sur le sujet, certains auteurs ont démontré que les conditions de développement (masse critique d'entreprises, infrastructure de soutien, synergies, etc.) étaient absentes dans les régions périphériques (Alesia, 2005; Morgan et Nauwelaers, 1999). Par ailleurs, Isaksen (2001) souligne que les régions périphériques ont peu de possibilités de stimuler l'innovation en 
raison de la difficulté à mettre en place des organismes avec lesquels les entreprises peuvent partager et échanger des connaissances.

En ce qui à trait aux pratiques d'innovation et à la compétitivité des entreprises du cluster maritime, nous observons que les stratégies compétitives en lien avec la qualité des produits et services offerts de même que les stratégies liées au respect des normes de qualité sont considérées par les entreprises comme étant les stratégies les plus importantes sur lesquelles elles définissent leur succès vis-à-vis leurs compétiteurs. Par ailleurs, la formation des employés de même que l'acquisition de machines et d'équipements sont les activités technologiques les plus fréquemment réalisées au sein des entreprises maritimes, devant les activités de R-D et l'achat d'expertise externe. Quant aux activités d'innovation, les entreprises maritimes sont peu innovantes. En effet, moins de $40 \%$ des entreprises enquêtées ont introduit des innovations de produits et procédés. Ce taux est considérablement inférieur à la moyenne régionale et nationale. De plus, les innovations introduites par les entreprises correspondent surtout à des innovations mineures, qui, bien que nouvelles pour l'entreprise, ne constituent pas de premières sectorielles ou mondiales. Pour ce qui est des partenariats, ceux-ci ont une importance mineure dans la réalisation des activités d'innovation des entreprises maritimes.

\subsection{Le système de soutien et l'offre de services spécialisés à l'industrie maritime}

Le système de soutien à l'industrie maritime regroupe l'ensemble des organisations, institutions et réseaux qui contribuent à l'évolution de la base de connaissance et qui alimentent les capacités et les dynamiques d'innovation au sein du cluster maritime. L'action de ces organisations vise à favoriser l'émergence de conditions nécessaires au développement industriel (présence de main-d'œuvre qualifiée, disponibilité d'une base de recherche fondamentale et appliquée, de capital-risque), à soutenir le développement des compétences de la main-d'œuvre, le développement industriel et l'entrepreneuriat. Pour atteindre ces objectifs, les organisations composant le système de soutien à l'industrie maritime offrent des services associés à différents domaines d'expertise : diffusion de l'information, formation spécialisée, soutien en recherche et développement, soutien financier, soutien en matière de technologies et pratiques de pointe, et services d'affaires. Les organisations comprises dans le système de soutien à l'industrie maritime sont présentées au tableau 1.

D'emblée, la quasi-totalité des organisations comprises dans le système de soutien sont issues de l'initiative publique. Certaines d'entre elles sont financées à $100 \%$ par le gouvernement, alors que d'autres doivent s'autofinancer en tout ou en partie. Le système de soutien à l'industrie maritime est composé de différentes constituantes. Ainsi, sur le territoire du Québec maritime se trouvent trois institutions d'enseignement et de formation liées au maritime (Université du Québec à Rimouski, Institut maritime du Québec, Centre spécialisé des pêches). En plus de participer au développement et au renouvellement des compétences dans les domaines des sciences et technologies marines, de la navigation, de l'aquaculture, des pêches et de la transformation des produits de la mer, ces institutions contribuent à la production, l'accumulation et la diffusion de savoirs associés 
Tableau 1 : Le système de soutien à l'industrie maritime

\begin{tabular}{|c|c|c|c|}
\hline Organisation* & Création & Employés & Activités principales \\
\hline \multicolumn{4}{|c|}{ Enseignement et formation } \\
\hline UQAR & 1969 & $\begin{array}{l}200 \text { professeurs et } \\
6000 \text { étudiants }\end{array}$ & $\begin{array}{l}\text { - Deux départements offrant des formations } \\
\text { spécialisées et multidisciplinaires dans le domaine } \\
\text { maritime }\end{array}$ \\
\hline IMQ & 1944 & $\begin{array}{l}175 \text { dont } 40 \\
\text { professeurs }\end{array}$ & $\begin{array}{l}\text { - Formations techniques dans la navigation, la } \\
\text { réparation navale, la plongée sous-marine et la } \\
\text { logistique de transport }\end{array}$ \\
\hline CSP & 1948 & $\begin{array}{l}57 \text { dont } 22 \\
\text { professeurs }\end{array}$ & $\begin{array}{l}\text { Formation collégiale en aquaculture et en } \\
\text { transformation des produits de la mer }\end{array}$ \\
\hline \multicolumn{4}{|c|}{ Instituts de recherche } \\
\hline ISMER & 1999 & $\begin{array}{l}22 \text { professeurs et } \\
150 \text { étudiants }\end{array}$ & $\begin{array}{l}\text { - Programmes de recherche et formation d'étudiants } \\
\text { gradués dans le domaine des sciences et } \\
\text { technologies marines }\end{array}$ \\
\hline IML & 1987 & 400 & $\begin{array}{l}\text { - Centre de recherche fédéral spécialisé en sciences } \\
\text { marines }\end{array}$ \\
\hline \multicolumn{4}{|c|}{ Sociétés de capital-risque } \\
\hline SODIM & 1997 & 7 & - Accompagnement et financement de capital-risque \\
\hline \multicolumn{4}{|c|}{ R-D et transfert technologique } \\
\hline IM & 2001 & 25 & $\begin{array}{l}\text { - CCTT en technologies maritimes dans la sécurité, } \\
\text { la plongée professionnelle, le transport et la gestion } \\
\text { maritime }\end{array}$ \\
\hline $\mathrm{CIDCO}$ & 2002 & 6 & - Application de méthodes et données géomatiques \\
\hline CRBM & 2004 & 29 & $\begin{array}{l}\text { - Centre de R-D et de transfert dans les } \\
\text { biotechnologies marines }\end{array}$ \\
\hline ССТTP & 1983 & 22 & $\begin{array}{l}\text { - CCTT dans le secteur des pêches, de l'aquaculture } \\
\text { et de la transformation }\end{array}$ \\
\hline CAM & 1992 & 20 & - Centre provincial de recherche en mariculture \\
\hline CTPA & 1969 & 14 & $\begin{array}{l}\text { - Centre provincial de recherche en transformation } \\
\text { des produits marins }\end{array}$ \\
\hline STMIM & 1980 & 15 & $\begin{array}{l}\text { Centre provincial de recherche dédié au } \\
\text { développement de la mariculture aux } \\
\text { Îles-de-la-Madeleine }\end{array}$ \\
\hline CATE & 2003 & 6 & $\begin{array}{l}\text { - Soutien au développement de nouveaux procédés } \\
\text { et produits dans les entreprises de pêche et de } \\
\text { transformation des produits de la mer }\end{array}$ \\
\hline $\mathrm{CACN}$ & 2005 & 4 & $\begin{array}{l}\text { - Centre de recherche privé dédié au développement } \\
\text { de la mariculture en Côte-Nord }\end{array}$ \\
\hline \multicolumn{4}{|c|}{ Développement des compétences de la main-d'œuvre } \\
\hline CSMOPM & 1997 & 7 & $\begin{array}{l}\text { Comité paritaire favorisant le développement } \\
\text { des compétences des entreprises de pêches, } \\
\text { d'aquaculture et de transformation }\end{array}$ \\
\hline RMQ & 1999 & 1 & $\begin{array}{l}\text { - Association vouée à la défense des intérêts des } \\
\text { entreprises maricoles }\end{array}$ \\
\hline \multicolumn{4}{|c|}{ Promotion de l'innovation } \\
\hline TMQ & 1999 & 3 & $\begin{array}{l}\text { - Réseau d'affaires regroupant les principaux } \\
\text { acteurs du secteur maritime chargé de favoriser le } \\
\text { développement du secteur et d'assurer la promotion } \\
\text { de celui-ci, au niveau national et international. }\end{array}$ \\
\hline
\end{tabular}

\footnotetext{
* Abréviations :

CACN : Centre aquacole de la Côte-Nord; CAM : Centre aquacole marin; CATE : Centre d'aide technologique aux entreprises; CCTT : Centres collégiaux de transfert technologique; CCTTP : Centre collégial de transfert technologique des pêches; CIDCO Centre interdisciplinaire de développement en cartographie des océans; CRBM : Centre de recherche sur les biotechnologies maritimes; CSMOPM : Comité sectoriel de la main-d'œuvre des pêches maritimes; CSP : Centre spécialisé des pêches; TMQ : Technopole maritime du Québec; CTPA : Centre technologique des produits aquatiques; IM : Innovation maritime; IML : Institut Maurice-Lamontagne; IMQ : Institut maritime du Québec; ISMER : Institut des sciences de la mer; RMQ : Regroupement des mariculteurs du Québec; SODIM : Société de développement de l'industrie maricole; STMIM : Station technologique maricole; UQAR : Université du Québec à Rimouski.
} 
à ces domaines. Le Québec maritime compte aussi deux instituts de recherche (Institut des sciences de la mer et Institut Maurice-Lamontagne) jouant un rôle important dans la production et la diffusion de connaissances fondamentales et appliquées dans différents domaines maritimes, notamment en matière d'océanographie côtière et de la gestion intégrée des écosystèmes. Les centres de R-D et de transfert technologique sont la composante du système de soutien à l'industrie maritime au sein de laquelle se retrouve le plus grand nombre d'organisations. Certains de ces centres possèdent une expérience particulière dans l'appui aux entreprises de l'aquaculture, des pêches et de la transformation des produits de la mer (Centre aquacole de la Côte-Nord, Centre aquacole marin, Centre d'aide technologique aux entreprises, Centres collégiaux de transfert technologique, Centre collégial de transfert technologique des pêches, Centre technologique des produits aquatiques, Station technologique maricole) alors que d'autres se spécialisent dans les domaines liés aux sciences et technologies marines (Innovation Maritime, Centre interdisciplinaire de développement en cartographie des océans, Centre de recherche sur les biotechnologies maritimes). Les sociétés de capital-risque constituent l'une des composantes les moins développées du système de soutien à l'industrie maritime. En effet, seule la Société de développement de l'industrie maricole offre du financement aux projets de démarrage, de diversification et d'expansion des entreprises maricoles. Parmi les autres composantes du système de soutien à l'industrie maritime se trouvent aussi deux organisations de développement des compétences de la main-d'œuvre (Comité sectoriel de la main-d'œuvre des pêches maritimes, Regroupement des mariculteurs du Québec) et une organisation de promotion de l'innovation (Technopole maritime du Québec).

Des entretiens réalisés avec les directeurs des organisations de soutien ont permis de faire ressortir certains facteurs limitant l'efficacité et l'efficience du système de soutien à l'industrie maritime :

- Peu de services d'affaires sont proposés. Alors qu'environ les deux tiers des organisations de soutien offrent des services dans le domaine de la diffusion d'information, de la recherche et développement et des technologies et pratiques de pointe, seulement deux organisations offrent des services d'affaires à l'industrie maritime. Pour favoriser l'émergence d'une masse critique et le développement des entreprises maritimes, ces services associés à l'organisation et au soutien à la production, à la gestion de la distribution, au conseil à l'exportation et à la commercialisation, à la recherche de nouveaux marchés, jouent un rôle primordial.

- Bien que certaines organisations aident les entreprises dans leurs recherches de financement (Technopole maritime du Québec, Institut maritime du Québec, et le Centre collégial de transfert technologique des pêches), et que d'autres encore offrent de manière occasionnelle des prêts ou des subventions (Centre d'aide aux entreprises de la CôteNord), les services de capital-risque sont au cœur de l'activité d'une seule organisation de soutien à l'industrie maritime, la Société de développement de l'industrie maricole, et le support offert par cette organisation est limité à l'industrie maricole. Étant donné le rôle structurant de cet organisme au sein de l'industrie maricole, il est possible de croire que l'absence d'une organisation offrant du capital-risque aux entreprises maritimes à caractère technologique constitue une faiblesse du système de soutien à l'industrie maritime.

- L'analyse des services destinés aux promoteurs de nouveaux projets industriels démontre que plusieurs services de soutien technologique sont disponibles (validation technologique, assistance à la R-D), mais que l'encadrement d'affaires spécifique à leurs besoins (accompa- 
gnement, suivi du projet), est limité, voire déficient. L'absence de structures d'intermédiation (parc scientifique et technologique, motel industriel, etc.) pour réunir et accueillir les entreprises maritimes en opération ou nouvellement créées est aussi à déplorer.

De plus, certaines conditions propres au financement public affectent de manière négative les organisations de soutien et les services qu'elles offrent auprès des entreprises maritimes :

- Dans le cadre de l'Initiative régionale stratégique, trois centres de R-D et de transfert technologique ont été créés. Ces organisations privées ont reçu du financement public pour procéder à l'acquisition et au développement de leurs infrastructures (laboratoires, locaux, équipement scientifique). Les ententes de financement avec les pouvoirs publics prévoient cependant qu'après une période de mise en marche, ces centres doivent s'autofinancer à des taux variés qui peuvent atteindre jusqu'à $90 \%$ du budget total d'opération. Cette contrainte a des répercussions majeures sur le développement et le fonctionnement de ces centres, mais surtout dans la qualité des services et dans la valorisation de leur compétence auprès des entreprises maritimes. Les centres collégiaux de transfert technologique, de même que la Technopole maritime du Québec, qui œuvre dans la promotion et l'animation du cluster maritime, vivent eux aussi ce problème de financement récurrent.

- Dans certaines organisations, particulièrement celles relevant directement du Gouvernement du Québec (Centre aquacole marin, Station technologique maricole, Centre technologique des produits aquatiques), les politiques internes font en sorte que les postes laissés vacants suite à des départs et mises à la retraite ne sont pas comblés. Étant donné la taille restreinte de ces organisations, la perte d'un expert sectoriel signifie bien souvent que l'organisme aura de la difficulté à répondre adéquatement aux demandes industrielles.

\subsection{Spécialisation et masse critique des acteurs et des réseaux du cluster maritime}

Les facteurs de développement et de succès des clusters sont étroitement liés à la présence d'une masse critique d'entreprises et d'institutions interconnectées œuvrant dans un domaine particulier (Martin et Sunley, 2004; Wolfe et Gertler, 2004; Wolfe et Lucas, 2004). Cette masse critique est associée à des économies d'agglomération qui favorisent l'accès aux clients, aux fournisseurs, aux services spécialisés, aux capitaux et à une maind'œuvre qualifiée, et par le fait même, contribue aussi à l'élévation de la productivité et à l'augmentation du degré d'innovation (Doloreux, 2004). Par ailleurs, Asheim et al. (2003) ont démontré que les politiques favorisant le développement de clusters peuvent viser à soutenir le développement d'une masse critique d'entreprises existantes ou encore à favoriser l'émergence d'une telle masse critique. Les mesures mises en place dans le cadre du projet de cluster maritime du Québec répondent à ce second objectif.

Pour ce qui est des entreprises maritimes existantes, nous retrouvons 96 entreprises actives dans l'une ou l'autre des six industries associées au cluster maritime. Certaines concentrations spatiales d'industries peuvent être observées. Ainsi, 13 des 16 entreprises actives en sciences et technologies marines sont situées au Bas-Saint-Laurent, principalement à Rimouski, alors que 35 des 49 entreprises œuvrant en aquaculture, en pêche et transformation des produits de la mer sont localisées en Gaspésie-Îles-de-laMadeleine, principalement dans la région de Gaspé. Seulement dix entreprises associées au cluster maritime sont localisées en Côte-Nord, et huit de celles-ci sont actives dans le domaine des pêches et de transformation des produits de la mer. 
La répartition spatiale et sectorielle des organisations de soutien à l'industrie maritime permet d'observer la présence de deux pôles distincts. Le premier, localisé à Rimouski, regroupe l'ensemble des organisations de soutien offrant des services aux entreprises technologiques de l'industrie maritime (biotechnologies maritimes, sciences et technologies marines). Le second, centré autour de Gaspé, mais possédant des ramifications aux Îles-de-la-Madeleine et en Côte-Nord, dessert les secteurs plus traditionnels (aquaculture, pêches et transformation des produits de la mer).

La présence simultanée d'une concentration d'entreprises et d'organisations de soutien œuvrant dans des domaines apparentés à Rimouski (sciences et technologies marines) et à Gaspé (aquaculture, pêches et transformation des produits de la mer) constitue l'une des bases sur lesquelles le développement du cluster maritime du Québec doit s'appuyer à moyen et long terme. En effet, étant donné la superficie du territoire compris dans le Québec maritime, pour favoriser l'émergence de masses critiques, il importe de mieux cibler les efforts de développement en tenant compte de la concentration et de la spécialisation des activités économiques et des organisations de soutien. Ces deux pôles sont cependant très différents l'un de l'autre.

Le pôle de Gaspé est orienté vers le marché. Cela est attribuable à la concentration d'entreprises de l'aquaculture, des pêches et de la transformation des produits de la mer autour de Gaspé et à la nature des services offerts par les organisations de soutien. En effet, l'ensemble du système de soutien est tourné vers le développement industriel de ces secteurs. Ainsi, les centres de R-D et de transfert technologique associés au pôle de Gaspé (Centre aquacole marin, Centre technologique des produits aquatiques, Station technologique maricole, Centre d'aide technologique aux entreprises, Centre aquacole de la Côte-Nord) offrent des services qui, bien que permettant le développement de nouveaux produits et processus, sont plus liés à la consolidation de l'industrie en place. En effet, ces centres œuvrant à l'amélioration et à l'adaptation de techniques existantes dans les domaines de l'aquaculture, des pêches et de la transformation des produits de la mer. Les services proposés par les autres organisations de ce pôle, c'est-à-dire la Société de développement de l'industrie maricole (capital-risque), le Comité sectoriel de la main-d'œuvre des pêches maritimes et le Regroupement des mariculteurs du Québec (développement des compétences) et le Centre spécialisé des pêches (formation technique) sont eux aussi axés vers le soutien au développement de l'industrie. En effet, ces organisations proposent du capital de risque, de l'accompagnement d'affaires et offrent des formations spécialisées, le tout afin de renforcer le système productif de l'aquaculture, des pêches et de la transformation des produits de la mer centré autour de Gaspé.

Le pôle de Rimouski est quant à lui orienté vers la recherche et le développement technologique dans le secteur des sciences et technologies marines. Il possède une composante institutionnelle importante qui influence grandement sa dynamique. En effet, Rimouski est le siège des deux seuls instituts de recherche du Québec maritime (Institut des sciences de la mer et Institut Maurice-Lamontagne), de la seule université du Québec maritime (Université du Québec à Rimouski) et de la seule institution d'enseignement québécoise dédiée au transport maritime (Institut maritime du Québec). Ces institutions de haut savoir contribuent aussi à enrichir la base de connaissance régionale. Elles contribuent aussi à mettre en place les conditions permettant le déve- 
loppement de l'industrie maritime, notamment en offrant une base de recherche importante et en contribuant au développement et au renouvellement des compétences de la main-d'œuvre spécialisée. Pour profiter des retombées localisées du savoir, des centres de R-D et de transfert technologique (Innovation maritime, Centre de recherche sur les biotechnologies maritimes et le Centre interdisciplinaire de développement en cartographie des océans) liés aux domaines d'activité des institutions d'enseignement et de formation et des instituts de recherche ont été créés dans le cadre du projet de cluster maritime. Ces centres visent à favoriser la transition d'un pôle institutionnel vers un pôle industriel. L'activité de Technopole maritime du Québec s'inscrit elle aussi dans cette logique de transition. En effet, cet organisme favorise l'appropriation des savoirs régionaux par les acteurs industriels et contribue à l'animation du milieu et au maillage entre les intervenants institutionnels et les entreprises maritimes.

\section{Implications des résultats de la recherche pour les politiques publiques}

L'objectif de cette section est de discuter de l'implication des résultats de cette recherche pour les politiques publiques. Des propositions générales de politiques sont présentées dans le but d'aider et de soutenir le développement potentiel du cluster maritime du Québec.

\subsection{Accrôtre l'innovation et la compétitivité des entreprises maritimes du cluster}

Afin de soutenir et d'aider les entreprises maritimes à être plus innovantes, les gouvernements doivent reconnaître la variété des besoins des entreprises des différents secteurs maritimes, car celles-ci possèdent des pratiques et des stratégies d'affaires différentes. De plus, ces entreprises ne sont pas toutes au même niveau de développement. Il est donc important de reconnaître ces différences afin de mieux définir et cibler les interventions selon la nature des besoins des entreprises des différents secteurs maritimes.

Par ailleurs, considérant que les entreprises maritimes offrent une gamme limitée de produits essentiellement destinés aux marchés locaux et provinciaux, et qu'elles sont peu exposées à la concurrence nationale et internationale, il est souhaitable de soutenir les entreprises maritimes dans la mise en place de stratégies de diversification visant le développement de produits uniques à forte valeur ajoutée. Par le développement de produits de niches, les entreprises maritimes pourront devenir plus compétitives. Pour ce faire, des interventions qui permettront d'augmenter le niveau de savoir incorporé dans la production et qui favoriseront l'acquisition et l'exploitation de nouvelles technologies permettront aux entreprises maritimes d'intégrer de nouvelles pratiques innovantes.

Finalement, la collaboration et le recours aux savoir-faire externes semblent essentiels aux entreprises pour innover et soutenir leur croissance. Il est donc souhaitable d'offrir une plus grande visibilité des activités réalisées et des technologies développées par les entreprises, les universités, les instituts de recherche, et les organisations de soutien du Québec maritime et d'ailleurs afin de permettre à l'industrie maritime de bénéficier du savoir-faire des autres membres du cluster, transformant ainsi des facteurs de concurrence en des arguments de partenariat. 


\subsection{L'offre de services spécialisés dédiés à l'industrie maritime}

Les gouvernements doivent poursuivre leur soutien financier aux organisations de soutien pour qu'elles puissent augmenter et enrichir leur niveau de compétences et ce afin de répondre adéquatement aux besoins des entreprises. Ce soutien, cependant, ne doit pas être ciblé seulement en phase de démarrage. À cet effet, il est crucial de fidéliser les programmes d'accompagnement dédiés à ces organisations. Ainsi, ces organisations pourront mettre en place une vision à moyen et long terme de transformation et de fonctionnement.

Au sein de l'offre de service, les services d'affaires et de commercialisation devraient jouer un rôle au moins aussi important que les services technologiques et scientifiques. Des infrastructures dédiées à leur accueil pourraient être favorables au développement de ces nouveaux projets, de même que l'offre de mesures de financement adaptées aux besoins des entreprises maritimes, et ce, à la fois pour soutenir les entreprises en phase de développement (pré-démarrage et démarrage) et les entreprises en croissance (R-D et commercialisation).

Finalement, dans le but d'augmenter le niveau de savoir-faire et les compétences des organisations de soutien, de même que les services spécialisés qu'elles peuvent offrir aux entreprises maritimes et autres, il est important pour les gouvernements de mettre à la disposition de ces organisations un fonds régional flexible visant à promouvoir la réalisation de recherches précompétitives aux effets structurants pour le développement de l'industrie maritime. Ce fonds pourrait être orienté vers la recherche appliquée de sorte à faire émerger des nouveaux projets industriels porteurs et novateurs dans l'industrie maritime.

\subsection{Spécialisation régionale et augmentation de la masse critique du cluster maritime du Québec}

Dans le cas du Québec maritime, nous pouvons distinguer un pôle à dominance technologique autour de Rimouski dans lequel les activités de recherche et développement dominent, et un pôle à dominance industrielle autour de Gaspé, davantage structuré autour de l'aquaculture, des pêches et de la transformation des produits de la mer. Le succès de ces pôles, indépendamment de leur orientation technologique ou productive, résultera de la combinaison sur un même territoire d'entreprises, de centres de formation et d'unités de recherche publiques ou privées engagées dans une synergie autour de projets communs au caractère innovant dans un domaine compétitif au niveau mondial.

Étant donné les différences majeures associées au développement de chacun de ces pôles - le pôle de Gaspé est résolument tourné vers le marché alors que le pôle de Rimouski tente de réussir sa transition d'un pôle institutionnel vers un pôle industriel basé sur le développement d'entreprises dans les sciences et technologies maritimes - les mesures et politiques destinées à favoriser l'émergence d'une masse critique au sein de chacun des deux pôles doivent être différenciées. Les mesures destinées au pôle de Gaspé doivent avoir pour finalité d'assurer la consolidation de l'industrie en place, c'est-à-dire d'aider les entreprises existantes à développer leurs marchés et à augmenter leur taille. Dans le cas de Rimouski, la transition d'un pôle institutionnel vers un pôle industriel passe obligatoirement par l'augmentation du nombre d'entreprises en sciences et technologies marines. Cette création d'entreprises peut être exogène (attraction 
d'entreprises existantes) ou endogène (développement de l'entrepreneuriat régional et de l'essaimage). Chacune de ces stratégies commande la mise en place de mesures et programmes différents et adaptés.

Compte tenu des différences sectorielles et régionales propres à la situation socioéconomique et à celle des entreprises maritimes, l'idée d'une politique ou d'une planification de développement uniformisée dans les régions du Bas-Saint-Laurent, de la GaspésieÎles-de-la-Madeleine, et de la Côte-Nord ne semble pas être la voie la plus appropriée en matière de développement de l'industrie maritime. Il est souhaitable de définir une démarche et une stratégie qui permettront de mieux tenir compte de ces différences régionales et sectorielles et d'appuyer les processus de développement des industries maritimes avec l'objectif d'insuffler dans chacun des pôles les éléments nécessaires à un cluster.

\section{Conclusion}

Bien que le développement de clusters véritables puisse prendre plusieurs décennies, comme ce fut le cas du cluster aéronautique à Montréal et du cluster des télécommunications à Ottawa, l'analyse du cluster maritime révèle l'absence d'éléments nécessaires à l'émergence des dynamiques propres aux clusters. En plus de l'absence d'une masse critique d'entreprises pionnières fondées sur des savoir-faire locaux spécifiques ou d'entreprises de l'extérieur, nous avons relevé l'absence ou la difficulté d'accès aux capitaux et à la technologie, la faible présence d'industries riches en savoir, et l'absence d'une infrastructure d'accueil pour les entreprises. Ceci n'est pas sans compter la distance qui sépare les membres du cluster et la distance éloignés des principaux marchés économique au Québec et d'ailleurs.

La combinaison des facteurs précédents explique qu'il est difficile de croire qu'une politique de développement basée sur les clusters, politique qui repose par définition sur la concentration géographique, sur un degré de proximité entre les acteurs et sur plusieurs concepts associés aux économies d'agglomération puisse fonctionner dans le Québec maritime. Cette région construite n'est pas équipée pour offrir la fréquence, le volume et la masse d'interactions requises entre les différents membres pour dynamiser ce cluster, ou du moins, le faire fonctionner. Cependant, la mise en place d'une stratégie qui favorisera le développement des deux pôles maritimes, en misant sur la mise en valeur des activités existantes à Rimouski et à Gaspé, semble une avenue de développement prometteuse afin de promouvoir et soutenir le développement de l'industrie maritime.

\section{Références}

Alasia, A., 2005. Compétences, innovation et croissance: les questions clés du développement rural et territorial - survol de la documentation, Ottawa.

Asheim, B.T, Isaksen, A., Nauwelaers, C. et Tödtling, F., 2003. Regional Innovation Policy for Small-Medium Enterprises. Edward Elgar, Cheltenham. 
Asheim, B.T. et Gertler, M. S., 2005. Regional Innovation Systems and the Geographical Foundations of Innovation. In: J. Fagerberg, D. Mowery and R. Nelson (Eds.), Oxford Handbook of Innovation. Oxford University Press, London.

Conseil national de recherche du Canada, 2005. Favoriser le développement des grappes technologiques au Canada. CNRC, Ottawa.

Cooke, P., Boekholt, P. et Tödtling, F., 2000. The Governance of Innovation in Europe: Regional Perspectives on Global Competitiveness. Pinter, London.

Développement économique Canada, 2005. Rapport sur les plans et les priorités 2005-2006. DEC, Montréal.

Doloreux, D., 2004. Regional innovation systems in Canada: a comparative study. Regional Studies, 38(5), 479-492.

Doloreux, D., 2006. Understanding regional innovation in the maritime industry: an empirical analysis. International Journal of Technology and Innovation Management, 3(2), 189-207.

Doloreux, D. et Shearmur, R., 2006. Regional development in sparsely populated areas: the case of the Quebec's missing maritime cluster. Canadian Journal of Regional Science Vol.29 (2). (sous presse).

European Commission, 2003. Thematic Report - Cluster Policies - Covering period up to March 2003. European Commission- Innovation/SMEs Programme, Luxembourg.

Industrie Canada, 2002. Atteindre l'excellence - la stratégie d'innovation du Canada. Industrie Canada, Ottawa.

IRE, 2006. An overview of Cluster Policies and Clusters in the New Member States of the European Union. IRE, Luxembourg.

Isaksen, A., 2001. Building regional innovation systems: is endogenous industrial development possible in the global economy? Canadian Journal of Regional Science, 24(1), 101-120.

Martin, R. et Sunley, P., 2003. Deconstructing Clusters: Chaotic Concept or Policy Panacea? Journal of Economic Geography, 3, 5-35.

Ministère des régions, 2002. Plein cap sur la mer! : stratégie québécoise de développement des ressources, sciences et technologies maritimes. Ministère des régions, Québec.

Ministère du développement économique, de l'innovation et de l'exportation, 2005. Les créneaux d'excellence des régions dans la compétition mondiale : le projet ACCORD. MDEIE, Québec.

Morgan, K. et Nauwelaers, C. (Eds.), 1999. Regional innovation strategies: the challenge for less-favoured regions. Routledge, London.

Nauwelaers, C. et Wintjes, R., 2002. Innovating SMEs and regions: the need for policy intelligence and interactive policies. Technology Analysis and Strategic Management, 14(2), 201-215.

OECD, 2001, Innovative Clusters: Drivers of National Innovation Systems. OECD, Paris.

Polèse, M. et R.Shearmur 2002. La périphérie et l'économie du savoir, (Montréal: INRS-Urbanisation, Culture et Société and Moncton: Institut Canadien de Recherche sur le Développement Régional)

Porter, M. E., 1990. L'avantage concurrentiel des nations. Éditions du Renouveau Pédagogique, Paris.

Porter, M.E., 2003. The economic performance of regions. Regional Studies, 37(6/7), 549-578.

Porter, M.E., 2004. Competitiveness in rural U.S. regions: learning and research agenda, Institute for Strategy and Competitiveness: Harvard Business School, Harvard.

Storper, M., 1997. The regional world. The Guillford Press, New York.

Wolfe, D. A. et Gertler, M. S., 2004. Clusters from the inside and out: local dynamics and global linkages. Urban Studies, 41(5/6), 1071-1093.

Wolfe, D. A. et Lucas, M., 2004. Clusters in a Cold Climate. McGill-Queen's University Press, Montreal \& Kingston. 